\title{
Binding of naturally occurring hydroxycinnamic acids to bovine serum albumin
}

\author{
Lucie Trnková $^{1,2}$, Iva Boušová ${ }^{\star *}$, Vladimír Kubíček $^{1}$, Jaroslav Dršata $^{1,2}$ \\ ${ }^{1}$ Charles University in Prague, Faculty of Pharmacy, Hradec Králové, Czech Republic; *Corresponding Author: Iva.Bousova@faf.cuni.cz \\ ${ }^{2}$ University of Hradec Králové, Faculty of Education, Hradec Králové, Czech Republic
}

Received 22 February 2010; revised 12 April 2010; accepted 13 May 2010.

\begin{abstract}
Hydroxycinnamic acids (HCAs) possess numerous biological effects including antioxidant, antiallergic, antimicrobial, and immunomodulatory activities and due to these properties are widely used in folk medicine. Nevertheless, they can interact with protein molecules and cause some structural and functional changes. The possibility of HCAs binding to bovine serum albumin (BSA) under physiological conditions was investigated by the UV-VIS absorption spectroscopy and fluorescence quenching method. Apart from rosmarinic acid, all tested HCAs quenched tryptophan fluorescence of BSA in the studied range of concentrations $(0-20 \mu \mathrm{M})$ mainly by static quenching mechanism (formation of nonfluorescent HCA-BSA complexes). The binding constants, number of binding sites and free energy changes were determined. The binding affinities of HCAs were ranked in the order: chlorogenic acid $>$ sinapic acid $\geq$ caffeic acid $>$ ferulic acid $>0$-coumaric acid $>p$-coumaric acid $\geq m$-coumaric acid, which was confirmed by spectral overlaps of BSA emission spectrum with absorption spectrum of HCA. All free energy changes possessed negative sign indicating the spontaneity of HCA-BSA interaction.
\end{abstract}

Keywords: Bovine Serum Albumin;

Hydroxycinnamic Acid; Fluorescence Quenching;

Protein-Ligand Binding

\section{INTRODUCTION}

Recently, considerable attention has been focused on the study of the interaction between small molecules (drugs) and biological macromolecules (e.g. proteins), especially discussing the thermodynamic quality, binding force quality, and mechanism of interactions [1-3]. These studies play crucial role in promoting research on proteins because they can provide useful information for study of pharmacological and biological effects of drugs as well as conformational changes of proteins caused by drugs.

Serum albumin is one of the most abundant proteins in circulatory system of a wide variety of organisms and one of the most extensively studied proteins at all $[4,5]$. Bovine serum albumin (BSA) consists of 583 amino acids in a single polypeptide chain cross-linked with 17 disulfide bonds. It is composed of three homologous domains (I-III), each of which comprises of two subdomains (A and B). BSA has two tryptophan residues, which significantly contribute to the intrinsic fluorescence of this protein: Trp-134 is located near the surface in domain IB and Trp-212 is buried in a hydrophobic (non-polar) pocket in the internal part of domain IIA [6]. HSA differs from BSA by $24 \%$ of primary structure and the most important difference from spectroscopic point of view seems to be presence of only one tryptophan residue (Trp-214) in its molecule [4,5]. Serum albumin possesses a wide range of physiological functions involving the binding, transport and deposition of many endogenous and exogenous ligands present in blood circulation $[4,7]$. Perhaps, its most outstanding property is the ability to bind a variety of ligands. It is well known that many drugs bind to serum albumin and their effectiveness depends on the binding ability [5,8]. On the other hand, drugs can cause various changes in protein conformation influencing its physiological function and such impaired proteins may be consequently pathologically accumulated in body tissues.

Plant polyphenols represent a heterogeneous group of natural compounds with one or more hydroxyl groups attached to the benzene ring. These substances possess several important physiological roles in plants, such as defense against herbivores and pathogens, pigmentation, and attraction of pollinating insects [9]. The most widely distributed polyphenolic compounds in plant tissues are hydroxycinnamic acids. Some of the most common 
naturally occurring HCAs are $p$-coumaric acid, ferulic acid, sinapic acid, and caffeic acid. These can be found in a free form but more often in various conjugated forms resulting from enzymatic hydroxylation, $O$-glycosylation, $O$-methylation or esterification [10,11]. Hydroxycinnamic acids have been reported to possess antimicrobial, antiallergic and anti-inflammatory activities, as well as antimutagenic and immunomodulatory effects $[12,13]$ and due to these properties are widely used in folk medicine. They exert also antioxidant and anti-radical activities [14-16]. Their biological effects are strongly dependent on the number and position of hydroxyl groups [15]. Just the presence of hydroxyl groups suggests the possibility of HCAs binding with molecules of proteins.

Spectroscopic techniques, such as ultraviolet-visible (UV-VIS) absorption spectroscopy [17], fluorescence spectroscopy [1,6], circular dichroism [18], and attenuated total reflectance-Fourier transform infrared spectroscopy [19] are commonly used tools to observe conformational changes in structure of proteins because of non-destructive measurements of substances in low concentration under physiological conditions, high sensitivity, rapidity and ease of implementation. Fluorescence spectroscopy is widely used to study mechanism of the binding between drugs and plasma proteins [6].

Nowadays, the investigation of the binding of naturally occurring polyphenolic compounds with various proteins attracts a great attention. Several spectroscopic studies on the interaction between bovine serum albumin and cinnamic acid [1], ferulic acid [20,21], chlorogenic acid [19,22] or various flavonoids [18,19,24] have been published. Also several studies dealing with the interaction of human serum albumin (HSA) with derivatives of cinnamic acid or flavonoids have been carried out $[2,3,17,25-29]$. The data obtained in several studies concerning the HCAs-BSA binding parameters (especially caffeic, chlorogenic, and ferulic acid) are hardly comparable because these studies were performed under various conditions (e.g. $\mathrm{pH}$, temperature).

The aim of the presented work was to study interactions of eight naturally occurring hydroxycinnamic acids with bovine serum albumin under physiological condi-

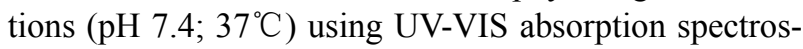
copy and fluorescence quenching method, reveal their character, evaluate structure-activity relationships, and compare obtained results with already published spectroscopic data on interaction of HCAs with BSA or HSA. The presented study contributes to the current knowledge in the area of protein-ligand binding, particularly bovine serum albumin-hydroxycinnamic acids interactions.

\section{EXPERIMENTAL}

\subsection{Chemicals}

Bovine serum albumin and all hydroxycinnamic acids were obtained from Sigma-Aldrich $\mathrm{GmbH}$, Germany. The chemical structures of tested HCAs are presented in Figure 1. All other chemicals were of analytical grade.

\subsection{Preparation of Stock Solutions}

Bovine serum albumin was dissolved in sodium phosphate buffer ( $\mathrm{pH} 7.4 ; 0.1 \mathrm{M} ; 0.05 \%$ sodium azide) in order to yield solutions with concentration $16 \mu \mathrm{M}$ and $2 \mu \mathrm{M}$ for UV-VIS absorption and fluorescence spectroscopic experiments, respectively. Individual HCAs were dissolved in anhydrous methanol in order to yield $10 \mathrm{mM}$ stock solutions. BSA and HCA solutions were prepared fresh before each measurement.

\subsection{UV-VIS Absorption Spectroscopy}

The UV-VIS spectra were recorded by a spectrophotometer Helios $\beta$ (Spectronic Unicam, United Kingdom) in a $10 \mathrm{~mm}$ quartz cuvette. Quantitative analysis of the potential interaction between HCAs and BSA was performed by the spectroscopic titration. Briefly, solution of BSA $(16 \mu \mathrm{M})$ was titrated in cuvette by successive additions of HCA solution $(10 \mathrm{mM})$ to a final concentration of $50 \mu \mathrm{M}$ (the drug to protein molar ratios were $0 ; 0.25$; $0.5 ; 0.75 ; 1.0 ; 1.25 ; 1.5 ; 1.75 ; 2.0 ; 2.5 ;$ and 3.125$)$ and the absorption spectra were recorded from 190 to 550 nm at $37^{\circ} \mathrm{C}$.

\subsection{Fluorescence Spectroscopy}

Fluorescence spectra were recorded using a luminescence spectrometer LS-50B (Perkin Elmer, United Kingdom) in a $10 \mathrm{~mm}$ quartz Suprasil fluorescence cuvette (Hellma,

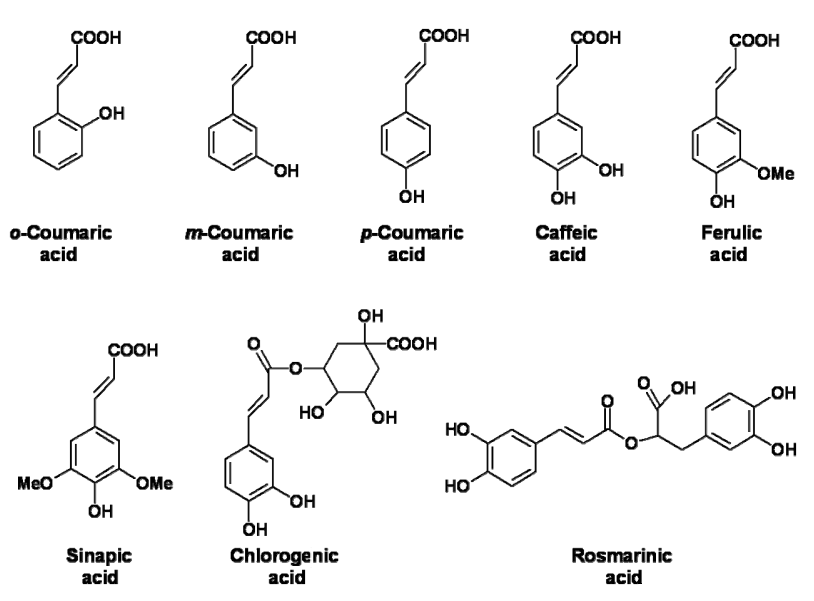

Figure 1. Chemical structures of tested hydroxycinnamic acids. 
Germany). Fluorescence emission spectra of individual HCA solutions $(25 \mu \mathrm{M})$ in sodium phosphate buffer $(\mathrm{pH}$ 7.4; $0.1 \mathrm{M} ; 0.05 \%$ sodium azide) were recorded. Quantitative analysis of the potential interaction between HCA and BSA was performed by the fluorimetric titration. Briefly, solution of BSA $(2 \mu \mathrm{M})$ was titrated in cuvette by successive additions of HCA solution (10 $\mathrm{mM}$ ) to a final concentration of $20 \mu \mathrm{M}$ (the drug to protein molar ratios were $0 ; 1.25 ; 2.5 ; 3.75 ; 5.0 ; 6.25 ; 7.5$; 8.75; and 10.0). Fluorescence emission spectra were recorded from 300 to $530 \mathrm{~nm}$ with excitation at $295 \mathrm{~nm}$ while stirring. The excitation and emission slits were both set to $5 \mathrm{~nm}$ and scanning speed to $200 \mathrm{~nm} / \mathrm{min}$. All experiments were carried out at $37^{\circ} \mathrm{C}$. Fluorescence intensity was read at emission wavelength of $350 \mathrm{~nm}$.

\subsection{Principles of Fluorescence Quenching}

The intensity of fluorescence can be decreased as a result of a wide variety of processes. Such declines in intensity are called quenching and can be caused by different molecular interactions. Dynamic quenching occurs when the excited-state fluorophore is deactivated upon contact with some other molecule (quencher) and no molecule is chemically altered during this process. In the case of static quenching, a non-fluorescent complex is formed between molecules of fluorophore and quencher. The static and dynamic quenching can be distinguished by the Stern-Volmer analysis. In the case the quenching is either purely static or dynamic, the plot shows linear dependence. When the plot of Stern-Volmer diagram shows exponential dependence, both static and dynamic quenching are exerted [6].

Dynamic quenching of fluorescence is described by the well-known Stern-Volmer equation as follows.

$$
F_{0} / F=1+k_{q} \tau_{0}[Q]=1+K_{D}[Q]
$$

In this equation, $F_{0}$ and $F$ are the fluorescence intensities of BSA in the absence and presence of quencher, respectively, $[Q]$ is the quencher concentration, $k_{q}$ is the bimolecular quenching constant, and $\tau_{0}$ is the lifetime of the fluorophore in the absence of quencher $\left(\tau_{0}\right.$ is about $5.10^{-9} \mathrm{~s}$, as to Reference [6]). The Stern-Volmer quenching constant is given by $k_{q} \tau_{0}$. In case the quenching is known to be dynamic, the Stern-Volmer constant will be presented by $\mathrm{K}_{\mathrm{D}}$ otherwise this constant will be described as $K_{S}[2,6]$. The dynamic quenching depends on diffusion, while static quenching does not. One criterion for distinguishing the type of quenching is the fact that the bimolecular quenching constant $k_{q}$ is larger than diffusion-limited rate constant of the biomolecule $\left(1 \times 10^{10}\right.$ $\mathrm{M}^{-1} \cdot \mathrm{s}^{-1}$ ) [6], so the static mechanism is the main reason that causes the fluorescence quenching (formation of a complex).
When small molecules bind independently to a set of equivalent sites on a macromolecule, the equilibrium between free and bound molecules is given by the equation:

$$
\log \left(F_{0}-F\right) / F=\log K_{b}+n \log [Q]
$$

where $K_{b}$ represents binding constant for quencher-protein interaction, $\mathrm{n}$ the number of binding sites per BSA, and $F_{0}, F$, have the same meaning as in (1) [2]. The values of $K_{b}$ and $\mathrm{n}$ could be determined from the intercept of y-axe and slope by plotting $\log \left(F_{0}-F\right) / F$ against log $[Q]$. Utilizing $K_{b}$, the free energy change $\left(\Delta G^{0}\right)$ value can be estimated from the following equation [30]:

$$
\Delta G^{0}=-R T \ln K_{b}
$$

The negative $\operatorname{sing} \Delta G^{0}$ value confirms the spontaneity of binding.

\section{RESULTS}

\subsection{Spectroscopic Study of Interactions between BSA and HCAs}

The UV-VIS absorption spectra of BSA titrated by individual HCAs solution were monitored in order to explore the structural changes of BSA caused by addition of these compounds. Spectral shifts were observed in all HCA-BSA systems with rising concentration of tested compound. Six HCAs ( $p$-coumaric, caffeic, ferulic, sinapic, chlorogenic, and rosmarinic acid) induced move of Trp absorption maximum $(280 \mathrm{~nm})$ to longer wavelengths which is called bathochromic (red) shift. Maximal spectral shift was about $6 \mathrm{~nm}$. The opposite phenomenon (blue shift) occurred in the absorption spectrum of BSA after interaction with $o$ - or $m$-coumaric acid. Absorbance maximum moved about $5 \mathrm{~nm}$ towards shorter wavelengths in both cases (data not shown).

\subsection{Fluorescence Quenching of BSA in the Presence of HCAs}

Quenching of protein intrinsic (tryptophan) fluorescence was employed for more detailed study of HCA-BSA binding. Fluorescence emission spectra were recorded upon excitation at $295 \mathrm{~nm}$, which is attributed to tryptophan residues only. Four individual HCAs (o-coumaric, caffeic, sinapic and ferulic acid) possessed remarkable emission maximum at 498, 432, 428 and $414 \mathrm{~nm}$, respectively. The most significant fluorescence intensity showed $o$-coumaric acid. Fluorescence intensities of HCA-BSA systems were read at emission wavelength of $350 \mathrm{~nm}$, where the emission maximum of BSA was located. Protein solution was titrated by successive additions of individual HCA solutions and its fluorescence 
intensity gradually decreased with rising concentration of HCA. This may indicate that the microenvironment around tryptophan residues in BSA molecule was altered due to the interaction with tested compound. Fluorescence emission spectrum of $p$-coumaric acid-BSA system is shown in Figure 2.

Red shifts of tryptophan emission maximum $(350 \mathrm{~nm})$ in dependence on increasing concentration of tested compounds were found in the case of $o$-coumaric, sinapic, chlorogenic, and rosmarinic acid. Emission maximum was slightly shifted towards longer wavelength by $2 \mathrm{~nm}$ for both $o$-coumaric and sinapic acid-BSA systems, and by 4 and $5 \mathrm{~nm}$ for chlorogenic and rosmarinic acid-BSA system, respectively. Other four tested HCAs did not cause any spectral shift. Emission spectra of $o$-coumaric, caffeic, ferulic, and sinapic acid involved isosbestic point, which might indicate that studied compounds exist both in bound and free form that are in equilibrium. The bound form exerts fluorescence whereas the unbound form does not (Figure 3).

It was noticed that emission spectra of these HCABSA systems above $430 \mathrm{~nm}$ corresponding with emission spectra of individual HCAs.

The type of fluorescence quenching of HCA-BSA systems was distinguished using the Stern-Volmer diagrams in the range of HCA concentrations of $0-20 \mu \mathrm{M}$. It was confirmed that the static quenching mechanism is the main reason of protein fluorescence quenching and consecutively the $\mathrm{K}_{\mathrm{S}}$ and $k_{q}(1)$ were determined from the slope of the linear regression curve of $F_{0} / F$ versus $[Q]$ (Table 1). The representative Stern-Volmer diagram of $o$-coumaric-BSA system is displayed in the inset of Figure 3. Rosmarinic acid exhibited exponential dependence (Figure 4) indicating that both types of quenching

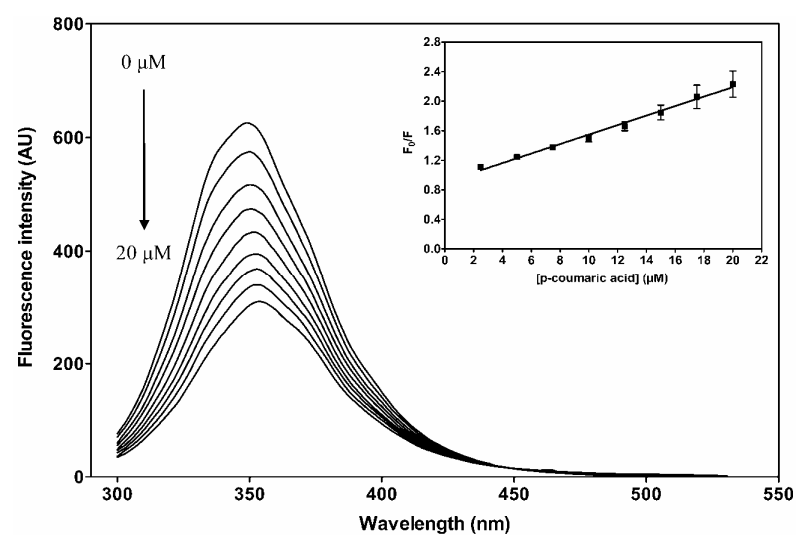

Figure 2. Fluorescence emission spectra of BSA $(2 \mu \mathrm{M})$ in the absence and in the presence of increasing amounts of $p$-coumaric acid $(0-20 \mu \mathrm{M})$ in sodium phosphate buffer $(\mathrm{pH} 7.4 ; 0.1 \mathrm{M}$; $0.05 \%$ sodium azide) at $\lambda_{\text {ex }}=295 \mathrm{~nm}$ and $37^{\circ} \mathrm{C}$. The inset shows the corresponding Stern-Volmer diagram of the $p$-coumaric acid-BSA system $\left(\lambda_{\mathrm{em}}=350 \mathrm{~nm}\right), \mathrm{R}^{2}=0.9908$.

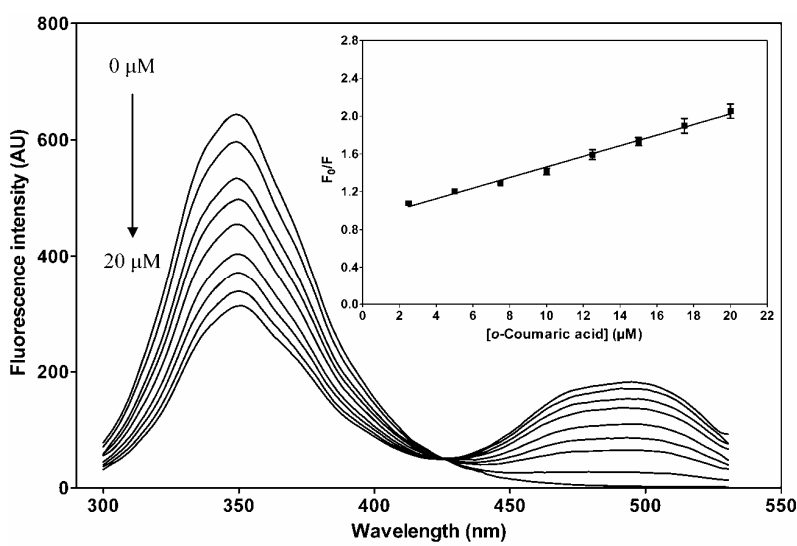

Figure 3. Fluorescence emission spectra of BSA $(2 \mu \mathrm{M})$ in the absence and in the presence of increasing amounts of $o$-coumaric acid $(0-20 \mu \mathrm{M})$ in sodium phosphate buffer $(\mathrm{pH} 7.4 ; 0.1$ $\mathrm{M} ; 0.05 \%$ sodium azide) at $\lambda_{\mathrm{ex}}=295 \mathrm{~nm}$ and $37^{\circ} \mathrm{C}$. The inset shows the corresponding Stern-Volmer diagram of the $o$-coumaric acid-BSA system $\left(\lambda_{\mathrm{em}}=350 \mathrm{~nm}\right), \mathrm{R}^{2}=0.9921$.

Table 1. The Stern-Volmer quenching constants $\left(\mathrm{K}_{\mathrm{S}}\right)$ and the bimolecular quenching constants $\left(k_{q}\right)$ of the system of HCABSA at $37^{\circ} \mathrm{C}$.

\begin{tabular}{lcc}
\hline Tested compound & $\begin{array}{c}\mathrm{K}_{\mathrm{S}} \pm \text { S.D. }{ }^{\mathrm{a}} \\
{\left[\times 10^{4} 1 . \mathrm{mol}^{-1}\right]}\end{array}$ & $\begin{array}{c}k_{q}^{\mathrm{b}} \pm \text { S.D. }{ }^{\mathrm{a}} \\
{\left[\times 10^{13} \mathrm{M}^{-1} \mathrm{~s}^{-1}\right]}\end{array}$ \\
\hline$o$-coumaric acid & $5.95 \pm 0.155$ & $1.19 \pm 0.031$ \\
$m$-coumaric acid & $5.96 \pm 0.170$ & $1.19 \pm 0.034$ \\
$p$-coumaric acid & $7.13 \pm 0.190$ & $1.43 \pm 0.038$ \\
caffeic acid & $4.30 \pm 0.263$ & $0.86 \pm 0.053$ \\
ferulic acid & $4.86 \pm 0.090$ & $0.97 \pm 0.018$ \\
sinapic acid & $4.25 \pm 0.209$ & $0.85 \pm 0.042$ \\
chlorogenic acid & $5.36 \pm 0.195$ & $1.07 \pm 0.039$ \\
\hline
\end{tabular}

${ }^{\mathrm{a}}$ standard deviation (mean value of three independent experiments); ${ }^{\mathrm{b}} k_{q}$ $=\mathrm{K}_{\mathrm{S}} / \tau_{0} ; \tau_{0}=5.10^{-9}$ s. [6]

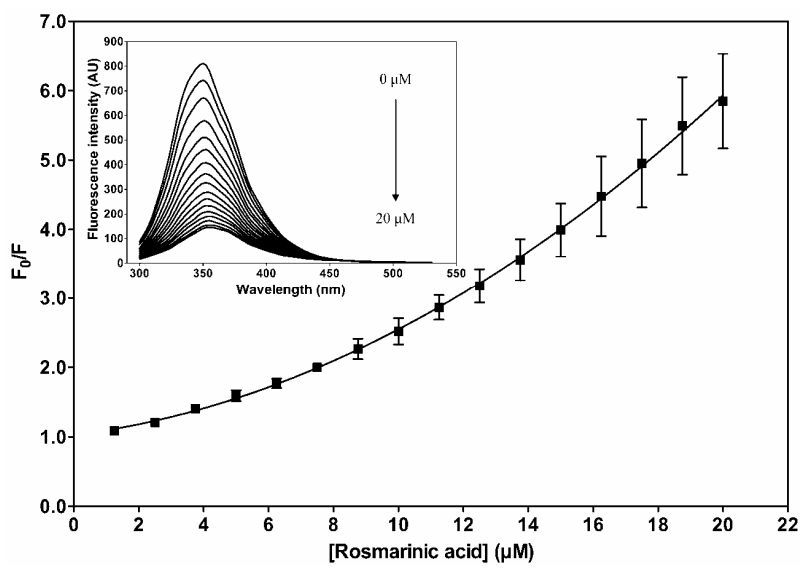

Figure 4. The Stern-Volmer diagram of the rosmarinic acidBSA system obtained by the titration with rosmarinic acid at $37^{\circ} \mathrm{C}$. [BSA] $=2 \mu \mathrm{M}$, [rosmarinic acid $]=0-20 \mu \mathrm{M}, \mathrm{pH} 7.4, \lambda_{\mathrm{ex}}=$ $295 \mathrm{~nm}, \lambda_{\text {em }}=350 \mathrm{~nm}$. The inset shows the corresponding fluorescence quenching spectra. 
were asserted and for this reason the $\mathrm{K}_{\mathrm{S}}\left(k_{q}\right)$ of the rosmarinic acid-BSA system was not determined.

It was noticed that emission spectra of these HCABSA systems above $430 \mathrm{~nm}$ corresponding with emission spectra of individual HCAs.

The type of fluorescence quenching of HCA-BSA systems was distinguished using the Stern-Volmer diagrams in the range of HCA concentrations of $0-20 \mu \mathrm{M}$. It was confirmed that the static quenching mechanism is the main reason of protein fluorescence quenching and consecutively the $\mathrm{K}_{\mathrm{S}}$ and $k_{q}(1)$ were determined from the slope of the linear regression curve of $F_{0} / F$ versus $[Q]$ (Table 1). The representative Stern-Volmer diagram of $o$-coumaric-BSA system is displayed in the inset of Figure 3. Rosmarinic acid exhibited exponential dependence (Figure 4) indicating that both types of quenching were asserted and for this reason the $\mathrm{K}_{\mathrm{S}}\left(k_{q}\right)$ of the rosmarinic acid-BSA system was not determined.

\subsection{Binding Parameters and Binding Mode of BSA-HCA Complexes}

Except for the rosmarinic acid-BSA system, the binding constants $\left(K_{b}\right)$, binding sites $(\mathrm{n})$, and free energy changes $\left(\Delta G^{0}\right)$ of all other HCA-BSA systems have been determined according to the Eqs. (2) and (3), respectively. Obtained values are presented in Table 2 and representative example of binding parameters determination for sinapic acid is displayed in Figure 5. The binding affinity was strongest for chlorogenic acid and ranked in the order chlorogenic acid $>$ sinapic acid $\geq$ caffeic acid $>$ ferulic acid $>o$-coumaric acid $>p$-coumaric acid $\geq$ $m$-coumaric acid. This order of binding affinities of HCA to BSA was confirmed also by spectral overlaps of BSA emission spectrum with absorption spectrum of individual HCAs. Example of spectral overlap for chlorogenic acid is shown in Figure 6. The negative

Table 2. The binding constants $\left(K_{b}\right)$, the number of binding sites (n) and the free energy change $\left(\Delta G^{0}\right)$ of the HCA-BSA system at $37^{\circ} \mathrm{C}$ which showed the static quenching mechanism.

\begin{tabular}{lccc}
\hline $\begin{array}{c}\text { Tested } \\
\text { compound }\end{array}$ & $\begin{array}{c}K_{b} \pm \mathrm{S} . D .^{\mathrm{a}} \\
{\left[\times 10^{5} 1 . \mathrm{mol}^{-1}\right]}\end{array}$ & $\mathrm{n} \pm$ S.D. ${ }^{\mathrm{a}}$ & $\begin{array}{c}\Delta G^{0} \pm \mathrm{S} . D .^{\mathrm{a}} \\
{\left[\mathrm{kJ} . \mathrm{mol}^{-1}\right]}\end{array}$ \\
\hline$o$-coumaric acid & $3.34 \pm 0.720$ & $1.17 \pm 0.012$ & $-32.73 \pm 0.563$ \\
$m$-coumaric acid & $1.31 \pm 0.045$ & $1.08 \pm 0.002$ & $-30.36 \pm 0.088$ \\
$p$-coumaric acid & $1.81 \pm 0.728$ & $1.10 \pm 0.027$ & $-30.98 \pm 1.096$ \\
caffeic acid & $4.16 \pm 1.659$ & $1.18 \pm 0.011$ & $-33.12 \pm 1.088$ \\
ferulic acid & $3.39 \pm 0.802$ & $1.18 \pm 0.019$ & $-32.75 \pm 0.621$ \\
sinapic acid & $4.19 \pm 0.117$ & $1.21 \pm 0.004$ & $-33.36 \pm 0.072$ \\
chlorogenic acid & $6.67 \pm 0.837$ & $1.23 \pm 0.016$ & $-34.55 \pm 0.325$ \\
\hline
\end{tabular}

${ }^{\mathrm{a}}$ standard deviation (mean value of three independent experiments)

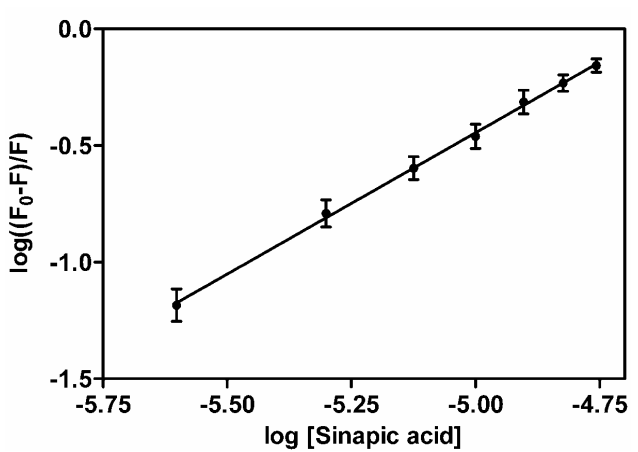

Figure 5. Logarithmic plots of fluorescence quenching of BSA treated with different concentrations of sinapic acid at physiological conditions $\left(37^{\circ} \mathrm{C} ; \mathrm{pH} 7.4\right)$. [BSA] $=2 \mu \mathrm{M}$, [sinapic acid] $=0-20 \mu \mathrm{M}, \lambda_{\mathrm{ex}}=295 \mathrm{~nm}$ and $\lambda_{\mathrm{em}}=350 \mathrm{~nm} . \mathrm{R}^{2}=0.9988$.

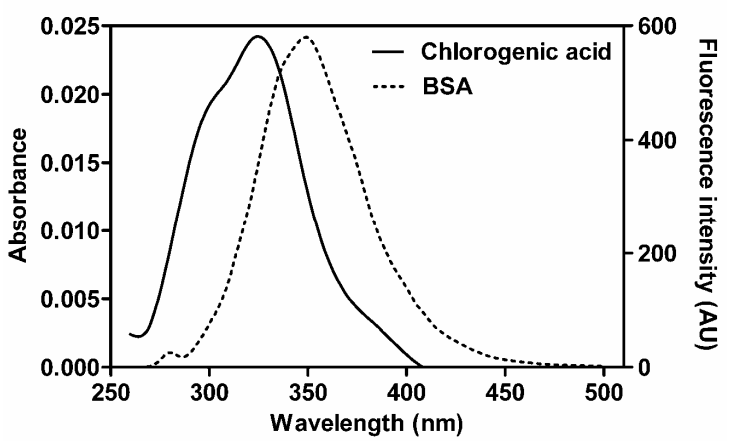

Figure 6. Overlap between the fluorescence emission spectrum of BSA and the absorption spectrum of chlorogenic acid at physiological conditions $\left(37^{\circ} \mathrm{C} ; \mathrm{pH} 7.4\right)$. [BSA] $=2 \mu \mathrm{M}$, [chlorogenic acid $]=2 \mu \mathrm{M}, \lambda_{\mathrm{ex}}=295 \mathrm{~nm}$ and $\lambda_{\mathrm{em}}=350 \mathrm{~nm}$.

value of $\Delta G^{0}$ indicating spontaneous process of HCABSA binding was determined for all studied interactions (Table 2).

\section{DISCUSSION}

Red shift in absorption maximum of tryptophan residues indicates changes in its microenvironment, where the polypeptide strand of BSA molecule is less extended and the hydrophobicity around Trp is increased. The conformational stability, rigidity, mechanical strength, and contributions of electrostatic interactions are enhanced by absence of water in the molecular interior [31]. On the other hand, blue shift implies that the BSA polypeptide strands are more extended and the hydrophocibity of Trp vicinity is decreased.

The changes in tryptophan microenvironment polarity are probably related to chemical structure of each HCA, namely to the number and position of hydroxyl groups. Hydrophobicity around tryptophan residues rose with increasing number of hydroxyl groups in the molecule of 
HCA. However, the position of hydroxyl groups on the benzene ring seemed to be also important. Only $o$ - and $m$-monosubstituted derivatives of cinnamic acid (o-coumaric and $m$-coumaric acid) caused increase in the polarity of Trp environment, while $p$-monosubstituted ( $p$ coumaric acid), di- and tri-substituted derivatives showed opposite effect.

Emission spectra of BSA were measured using excitation wavelength at $295 \mathrm{~nm}$ to ensure that the light caused excitation only of tryptophan residues. These are highly susceptible to any change in their local environment resulting in appearance of a substantial spectral shift [6]. In contrast to absorption spectroscopy, the red shift of Trp emission band is caused by decrease in hydrophobic property of its environment in protein molecule suggesting that tryptophan residue has been brought to more hydrophilic environment [3] and protein secondary structure has been changed [26].

Red shift in emission spectra of four studied hydroxycinnamic acids (o-coumaric, sinapic, chlorogenic, and rosmarinic acid), which indicated that binding of these compounds to BSA was associated with changes in the dielectric environment of at least one of its two indole rings, was observed. Only slight blue shift and no shift were noticed during interaction of cinnamic acid with BSA and HSA, respectively [1,2]. It can be expected that changes found in emission spectra of HCA-BSA systems were connected with alterations in microenvironment of Trp-134 because Trp-212 in BSA molecule is in the similar position as Trp-214, where cinnamic acid caused no spectral shift of its emission band. Increasing number of hydroxyl groups in the molecule of HCA was accompanied by decline of hydrophobicity around Trp. The significant changes in BSA emission spectrum were observed after its interaction with chlorogenic or rosmarinic acid. Other authors described red shifts by 3 to 18 $\mathrm{nm}$ in HSA spectrum upon reaction with $p$-coumaric, ferulic, sinapic, and chlorogenic acid $[2,3,25]$ but the concentrations applied in these experiments were higher (up to $150 \mu \mathrm{M}$ ) than those used in presented work.

The natural lifetime for the biological macromolecules $\left(\tau_{0}\right)$ is generally given as $10^{-8} \mathrm{~s}[1,3]$. However, the value for BSA is more precisely estimated as $5 \times 10^{-9} \mathrm{~s}$ [6]. The latter mentioned value was used in this study for calculation of bimolecular quenching constants $\left(k_{q}\right)$ which reflect efficiency of quenching or the accessibility of the fluorophores to the quencher. The fact that the value of $k_{q}$ is higher than the value of the diffusion-limited rate constant of the biomolecule $\left(1 \times 10^{10}\right.$ $\left.\mathrm{M}^{-1} \cdot \mathrm{s}^{-1}\right)$ is one of the criterions for determination of static mechanism of quenching $[1,6]$.

Only small but significant differences among $k_{q}$ values for the tested HCA-BSA systems were observed. All tested HCAs exerted better quenching effect than cinnamic acid $\left(2.26 \times 10^{12} \mathrm{M}^{-1} \cdot \mathrm{s}^{-1}\right.$ for $\left.\tau_{0}=10^{-8} \mathrm{~s}\right)$ [1]. Rosmarinic acid caused the most outstanding decrease in fluorescence intensity of BSA in the range of studied concentrations but it exhibited exponential dependence in the Stern-Volmer diagram and thus was not considered in overall comparison. Coumaric acids showed stronger quenching activity than the other more substituted HCAs. This effect was probably dependent upon the position of hydroxyl group on aromatic ring and $p$-position was determined as the most suitable location of hydroxyl group. The $k_{q}$ value of chlorogenic acid was similar to $k_{q}$ of coumaric acids and slightly higher than $k_{q}$ of more substituted derivatives (Table 1). It was probably caused by the presence of five hydroxyl groups in its molecule and their spatial arrangement [3]. The lowest quenching effect was observed for sinapic and caffeic acid followed by ferulic acid. Presence of methoxy group seemed to be important for quenching activity too. Ferulic acid showed higher effect than caffeic acid, while the $k_{q}$ of sinapic acid was slightly lower compared to caffeic acid. This was perhaps caused by steric hindrance in molecule of sinapic acid. The obtained bimolecular quenching constant for chlorogenic acid-BSA system is in good agreement with data found in literature [19]. However, published data are inconsistent and even in one case no quenching by chlorogenic acid was observed [20]. More studies were published for some HCA-HSA systems. The highest $k_{q}$ was obtained for chlorogenic acid followed by caffeic and sinapic, while ferulic and $p$-coumaric acid possessed lower $k_{q}$ values. Data obtained for HCA-BSA systems in this study corresponded with these findings by other authors $[2,3]$.

In general, the binding constant $K_{b}$ reflects the power of ligand-protein association and thus can be used for comparison of binding affinities of structurally-related ligands to protein molecule connected with alterations of its secondary structure. Number of binding sites is another important parameter that contributes to better understanding of ligand-protein interaction [1-3].

The binding constant $K_{b}$ for cinnamic acid-BSA system mentioned in the literature [1,2] is lower than values obtained in our experiments for HCA-BSA systems, which may confirm significance of hydroxyl groups in the process of binding. Moreover, binding affinity of cinnamic acid is higher for BSA than for HSA, which indicates that also binding of HCAs to BSA may be more pronounced [2]. It was demonstrated that interaction of HCAs with protein molecule depends mainly on the size and structure of ligand, especially on the number and position of hydroxyl groups on the aromatic ring [20,32]. Hydroxyl groups of studied compounds form hydrogen bonds with amino acid residues in the protein 
molecule. Another important factor influencing ligandprotein binding is aromaticity of the ligand molecule because hydrophobic interactions are formed between aromatic rings of ligand and amino acid residues [25]. Chlorogenic acid with two aromatic hydroxyls and three hydroxyls on cyclohexane ring exerted the strongest binding affinity because this compound can form hydrogen bonds with protein more easily than other less-substituted HCAs, e.g. ferulic acid. Similar results were published also for ferulic acid-HSA and chlorogenic acid-HSA system [3]. It is evident that the hydroxyls substituted on aromatic ring of the HCAs play an important role in the changes of BSA secondary structure. Monosubstituted HCAs possessed the lowest binding affinities of all HCAs studied. The differences among binding affinities of sinapic, ferulic and caffeic acid were not statistically significant.

The binding parameters of chlorogenic and ferulic acid with bovine serum albumin have been intensively studied by other authors, while no information about other HCAs was found in the literature. The value of $K_{b}$ reported by Tang et al. [19] for chlorogenic acid-BSA system using fluorescence quenching method is lower than value obtained in performed experiments, but the authors used higher concentrations of chlorogenic acid and different experimental conditions. Moreover, Rawel et al. [20,21] reported that chlorogenic acid does not quench Trp fluorescence in BSA and determined its binding constant by Hummel-Dreyer/size exclusion chromatography which showed significantly lower value of $K_{b}$ in comparison with results presented by Tang et al. [19]. Non-covalent interactions of chlorogenic acid with BSA have been studied by Prigent et al. [33], who reported that these interactions decrease with the increasing temperature while $\mathrm{pH}$ and ionic strength had no pronounced effect. Zhang et al. [34] reported the $K_{b}$ for ferulic acid-BSA system determined by affinity capillary electrophoresis which is in good agreement with our result. By contrast, Rawel et al. [20] determined binding constant of ferulic acid-BSA system by fluorescence quenching method and Hummel-Dreyer/size exclusion chromatography, where first method gave similar results to our data and $K_{b}$ obtained by second method was significantly lower. However, several studies dealing with binding of $p$-coumaric, caffeic, ferulic, sinapic acid, and chlorogenic acid with HSA have been published $[2,3,25]$.

The results showed that the numbers of binding sites ranged between 1.08 and 1.23 suggesting that one molecule of BSA was associated with one molecule of HCA in the drug to protein ratio up to 10 for the tested HCAs apart. The number of binding sites rose with increasing number of hydroxyl groups in the ligand molecule.
The free enthalpy had negative sign for all studied interactions which indicates the spontaneity of the interaction between BSA and hydroxycinnamic acids. The relatively strong binding enthalpy underlines the stability of BSA-HCA complexes from the energetic point of view. These findings are supported by data found in literature $[1,20,21]$.

\section{CONCLUSIONS}

Apart from rosmarinic acid, all tested HCAs quenched tryptophan fluorescence of BSA in the studied range of concentrations $(0-20 \mu \mathrm{M})$ mainly by static quenching mechanism and thus showed the formation of non-fluorescent HCA-BSA complexes. For this reason the rosmarinic acid-BSA system was not concluded in the overall assessment of binding affinities. The obtained results suggest that the binding affinity and number of binding sites depend on the number and position of hydroxyl groups in the molecule of HCA. Disubstituted and trisubstituted derivatives exhibited stronger binding affinity than monosubstituted derivatives. The number of binding sites for all HCAs ranged from 1.08 to 1.23 suggesting that one molecule of BSA associates with one molecule of HCA. All HCA-BSA interactions were spontaneous processes based on $\Delta G^{0}$. The results imply that HCAs could be stored and transported in blood by serum albumin which may influence their biological and pharmacological activities. On the other hand, physiological functions of this protein could be altered by ligand binding.

\section{ACKNOWLEDGEMENTS}

The presented study was supported by the Specific research 2009 of the University of Hradec Králové.

\section{REFERENCES}

[1] Bian, H., Zhang, H., Yu, Q., Chen, Z. and Liang, H. (2007) Studies on the interaction of cinnamic acid with bovine serum albumin. Chemical \& Pharmaceutical Bulletin, 55(6), 871-875.

[2] Jiang, M., Xie, M.X., Zheng, D., Liu, Z., Li, X.Y. and Chen, X. (2004) Spectroscopic studies on the interaction of cinnamic acid and its hydroxyl derivatives with human serum albumin. Journal of Molecular Structure, 692(1-2), 71-80.

[3] Kang, J., Liu, Y., Xie, M.X., Li, S., Jiang, M. and Wang, Y.D. (2004) Interactions of human serum albumin with chlorogenic acid and ferulic acid. Biochimica et Biophysica Acta, 1674(2), 205-214.

[4] Peters, T. (1996) All about albumin: Biochemistry, genetics, and medical applications. Academic Press, San Diego. 
[5] Carter, D.C. and Ho, J.X. (1994) Structure of serum albumin. Advances in Protein Chemistry, 45, 153-203.

[6] Lakowicz, J. R. (2004) Principles of fluorescence spectroscopy. 2nd Edition, Springer, New York.

[7] Behrens, P.Q., Spiekerman, A.M. and Brown, J.R. (1975) Structure of bovine serum-albumin. Federation Proceedings, 34(3), 591.

[8] Kragh-Hansen, U. (1981) Molecular aspects of ligand binding to serum albumin. Pharmacological Reviews, 33(1), 17-53.

[9] Dewick, P. M. (2002) Medicinal natural products: A biosynthetic approach. 2nd Edition, Wiley, Chichester.

[10] Harborne, J.B. and Williams, C.A. (2000) Advances in flavonoid research since 1992. Phytochemistry, 55(6), 481-504.

[11] Rice-Evans, C.A., Miller, N. and Paganga, G. (1997) Antioxidant properties of phenolic compounds. Trends in Plant Science, 2(4), 152-159.

[12] Natarajan, K., Singh, S., Burke, T.R., Grunberger, D. and Aggarwal, B.B. (1996) Caffeic acid phenethyl ester is a potent and specific inhibitor of activation of nuclear transcription factor NF-kappa B. Proceedings of the $\mathrm{Na}$ tional Academy of Sciences, USA, 93(17), 9090-9095.

[13] Pannala, A.S., Razaq, R., Halliwell, B., Singh, S. and Rice-Evans, C.A. (1998) Inhibition of peroxynitrite dependent tyrosine nitration by hydroxycinnamates: Nitration or electron donation? Free Radical Biology and Medicine, 24(4), 594-606.

[14] Natella, F., Nardini, M., Di Felice, M. and Scaccini, C.J. (1999) Benzoic and cinnamic acid derivatives as antioxidants: Structure-activity relation. Journal of Agricultural and Food Chemistry, 47(4), 1453-1459.

[15] Rice-Evans, C.A., Miller, N.J. and Paganga, G. (1996) Structure-antioxidant activity relationships of flavonoids and phenolic acids. Free Radical Biology and Medicine, 20(7), 933-956.

[16] Graf, E. (1992) Antioxidant potential of ferulic acid. Free Radical Biology and Medicine, 13(4), 435-448.

[17] Kanakis, C.D., Tarantilis, P.A., Polissiou, M.G., Diamantoglou, S. and Tajmir-Riahi, H.A. (2006) Antioxidant flavonoids bind human serum albumin. Journal of Molecular Structure, 798(1-3), 67-74.

[18] Tian, J., Liu, J., Hu, Z. and Chen, X. (2005) Interaction of wogonin with bovine serum albumin. Bioorganic \& Medicinal Chemistry, 13(12), 4124-4129.

[19] Tang, D., Li, H.J., Wen, X.D. and Qian, Z.M. (2008) Interaction of bioactive components caffeoylquinic acid derivatives in chinese medicines with bovine serum albumin. Chemical \& Pharmaceutical Bulletin, 56(3), 360-365.

[20] Rawel, H.M, Frey, S.K., Meidtner, K., Kroll, J. and Schweigert, F.J. (2006) Determining the binding affinities of phenolic compounds to proteins by quenching of the intrinsic tryptophan fluorescence. Molecular Nutrition \& Food Research, 50(8), 705-713.

[21] Rawel, H.M, Meidtner, K. and Kroll, J. (2005) Binding of selected phenolic compounds to proteins. Journal of Agricultural and Food Chemistry, 53(10), 4228-4235.

[22] Rawel, H.M, Rohn, S., Kruse, H.P. and Kroll, J. (2002) Structural changes induced in bovine serum albumin by covalent attachment of chlorogenic acid. Food Chemistry, 78(4), 443-455.

[23] Tian, J., Liu, J., Tian, X., Hu, Z. and Chen, X. (2004) Study of the interaction of kaempferol with bovine serum albumin. Journal of Molecular Structure, 691(1-3), 197202.

[24] Papadopoulou, A., Green, R.J. and Frazier, R.A. (2005) Interaction of flavonoids with bovine serum Albumin: A fluorescence quenching study. Journal of Agricultural and Food Chemistry, 53(1), 158-163.

[25] Liu, Y., Xie, M.X., Jiang, M. and Wang, Y.D. (2005) Spectroscopic investigation of the interaction between human serum albumin and three organic acids. Spectrochimica Acta Part A, 61(9), 2245-2251.

[26] He, W., Li, Y., Xue, C., Hu, Z., Chen, X. and Sheng, F. (2005) Effect of Chinese medicine alpinetin on the structure of human serum albumin. Bioorganic \& $\mathrm{Me}$ dicinal Chemistry, 13(5), 1837-1845.

[27] Xie, M.X., Long, M., Liu, Y., Qin, C. and Wang, Y.D. (2006) Characterization of the interaction between human serum albumin and morin. Biochimica et Biophysica Acta, 1760(8), 1184-1191.

[28] Xie, M.X., Xu, X.Y. and Wang, Y.D. (2005) Interaction between hesperetin and human serum albumin revealed by spectroscopic methods. Biochimica et Biophysica Acta, 1724(1-2), 215-224.

[29] Liu, J., Tian, J., Li, Y., Yao, X., Hu, Z. and Chen, X. (2004) Binding of the bioactive component daphnetin to human serum albumin demonstrated using tryptophan fluorescence quenching. Macromolecular Bioscience, 4(5), 520-525.

[30] Shang, L., Jiang, X. and Dong, S. (2006) In vitro study on the binding of neutral red to bovine serum albumin by molecular spectroscopy. Journal of Photochemistry and Photobiology A, 184(1-2), 93-97.

[31] Hu, Y.J., Liu, Y., Zhao, R.M., Dong, J.X. and Qu, S.S. (2006) Spectroscopic studies on the interaction between methylene blue and bovine serum albumin. Journal of Photochemistry and Photobiology A, 179(3), 324-329.

[32] Bartolome, B., Estrella, I. and Hernandez, M.T. (2000) Interaction of low molecular weight phenolics with proteins (BSA). Journal of Food Science, 65(4), 617-621.

[33] Prigent, S.V.E., Gruppen, H., Visser, A.J.W.G., van Koningsveld, G.A., de Jong, G.A.H. and Voragen, A.G.J. (2003) Effects of non-covalent interactions with 5-Ocaffeoylquinic acid (chlorogenic acid) on the heat denaturation and solubility of globular proteins. Journal of Agricultural and Food Chemistry, 51(17), 5088-5095.

[34] Zhang, Y., Xu, M., Du, M. and Zhou, F. (2007) Comparative studies of the interaction between ferulic acid and bovine serum albumin by ACE and surface plasmon resonance. Electrophoresis, 28(11), 1839-1845. 